\title{
Poorly-Controlled Type 1 Diabetes Mellitus Impairs LH-LHCGR Signaling in the Ovaries and Decreases Female Fertility in Mice
}

\author{
Jaewang Lee ${ }^{1,2 *}$, Hoi Chang Lee ${ }^{2 *}$, So-Youn $\mathrm{Kim}^{3}$, Geum Joon $\mathrm{Cho}^{2,4}$, and Teresa K. Woodruff ${ }^{2}$ \\ ${ }^{1}$ Department of Biomedical Laboratory Science, College of Health Science, Eulji University, Seongnam, Korea; \\ ${ }^{2}$ Department of Obstetrics and Gynecology, Feinberg School of Medicine, Northwestern University, Chicago, IL, USA; \\ ${ }^{3}$ Olson Center for Women's Health, Department of Obstetrics and Gynecology, and Fred and Pamela Buffett Cancer Center, \\ University of Nebraska Medical Center, Omaha, NE, USA; \\ ${ }^{4}$ Department of Obstetrics and Gynecology, Korea University College of Medicine, Seoul, Korea.
}

Purpose: The aim of this study was to investigate how type I diabetes mellitus (T1D) affects the folliculogenesis and oocyte development, fertilization, and embryo development.

Materials and Methods: A comparative animal study was conducted using two different mouse models of T1D, a genetic AKITA model and a streptozotocin-induced diabetes model. Ovarian function was assessed by gross observation, immunoblot, immunohistochemistry, oocyte counting, and ELISA for serum hormones (insulin, anti-Mullerian hormone, estradiol, testosterone, and progesterone). Maturation and developmental competence of metaphase II oocytes from control and T1D animals was evaluated by immunofluorescent and immunohistochemical detection of biomarkers and in vitro fertilization.

Results: Animals from both T1D models showed increased blood glucose levels, while only streptozotocin (STZ)-injected mice showed reduced body weight. Folliculogenesis, oogenesis, and preimplantation embryogenesis were impaired in both T1D mouse models. Interestingly, exogenous streptozotocin injection to induce T1D led to marked decreases in ovary size, expression of luteinizing hormone/chorionic gonadotropin receptor in the ovaries, the number of corpora lutea per ovary, oocyte maturation, and serum progesterone levels. Both TID models exhibited significantly reduced pre-implantation embryo quality compared with controls. There was no significant difference in embryo quality between STZ-injected and AKITA diabetic mice.

Conclusion: These results suggest that T1D affects folliculogenesis, oogenesis, and embryo development in mice. However, the physiological mechanisms underlying the observed reproductive effects of diabetes need to be further investigated.

Key Words: Type 1 diabetes mellitus, reproduction, folliculogenesis, oocyte, signaling

\section{INTRODUCTION}

Type 1 diabetes mellitus (T1D) occurs as a result of autoimmune

Received: February 22, 2019 Revised: April 23, 2019

Accepted: May 7, 2019

Corresponding author: Teresa K. Woodruff, PhD, Department of Obstetrics and Gynecology, Feinberg School of Medicine, Northwestern University, 303 E. Superior Street, Lurie 10-250, Chicago, IL 60611, USA.

Tel: 1-312-503-2504, Fax: 1-312-503-2019, E-mail: tkw@northwestern.edu

* Jaewang Lee and Hoi Chang Lee equally contributed to this work.

- The authors have no potential conflicts of interest to disclose.

(c) Copyright: Yonsei University College of Medicine 2019

This is an Open Access article distributed under the terms of the Creative Commons Attribution Non-Commercial License (https://creativecommons.org/licenses/ by-nc/4.0) which permits unrestricted non-commercial use, distribution, and reproduction in any medium, provided the original work is properly cited. destruction of beta cells in the pancreas, leading to a loss of insulin production and hyperglycemia. ${ }^{1}$ The causes of T1D are not completely clear and may be genetic or environmental, such as viral infection or exposure to toxic chemicals. ${ }^{2}$ Women who are positive for auto-antibodies, such as antibodies to insulin, glutamic acid decarboxylase, protein tyrosine phosphatase, and others, are at high risk of developing T1D. ${ }^{3}$ Poorly controlled T1D can have severe effects on female reproductive health, including low fertility, hypogonadism through impairment of the hypothalamic-pituitary-ovarian (HPO) axis, miscarriage, and congenital malformation. Female mouse models of T1D show hypogonadism, reduced ovary size, and decreased oocyte quality. ${ }^{4,5}$ Within the ovarian follicle, T1D mice show impaired granulosa cell-oocyte communication, mito- 
chondrial dysfunction during meiosis, apoptosis of cumulus cells, and alteration of DNA methylation status..$^{6-9}$ Nevertheless, the mechanisms underlying these effects of diabetes on reproduction are not completely understood.

Most studies of the reproductive effects of T1D have used a single animal model, either a genetic- or chemical-induced model of T1D. The AKITA mouse expresses an autosomal dominant mutation on chromosome 7 that leads to a cysteine to tyrosine change and misfolding of the insulin 2 protein. ${ }^{10}$ This induces abnormal activation of the unfolded protein response, which causes endoplasmic reticulum (ER) stress and apoptosis of pancreatic beta cells. ${ }^{11,12}$ Streptozotocin (STZ) is a toxic glucose analogue that accumulates in pancreatic beta cells through glucose transporter- 2 and causes selective cell death. ${ }^{13,14}$ Injections of high doses of STZ are used to chemically induce TID in mice. In both genetic and chemically induced mouse models of T1D, the destruction of pancreatic beta cells leads to hypoinsulinemia and the inability to regulate blood glucose.

To shed light on the underlying mechanisms driving impaired fertility in women with T1D, we sought to compare the effects of T1D on a comprehensive set of female ovarian functional endpoints-from follicle growth to oocyte maturation to embryo development-in two mouse models. We hypothesized that T1D caused by either a genetic mutation (AKITA model) or an environmental factor (STZ-induced model) would affect ovarian physiology.

\section{MATERIALS AND METHODS}

\section{Animals}

The use of mouse models of T1D in this study was approved by the Institutional Animal Care and Use Committee (IACUC) of Northwestern University (IS00000442). All procedures were carried out under an IACUC-approved protocol. Animals were housed in a temperature- and light-controlled environment (14h light: 10h dark) and provided with food and water $a d l i$ bitum. Animals were fed Teklad Global irradiated 2919 chow, which does not contain soybean or alfalfa meal and contains minimal phytoestrogens as described in a previous paper. ${ }^{15}$ Heterozygous AKITA male (003548, The Jackson Laboratory, Bar Harbor, ME, USA) and wild type C57BL/6J female (000664, The Jackson Laboratory, Bar Harbor, ME, USA) mice were bred, and the offspring were genotyped to confirm AKITA heterozygosity. For the STZ model of T1D, wild-type C57BL/6J mice were administered $190 \mathrm{mg} / \mathrm{kg}$ of STZ (S0130-1G, MilliporeSigma, Burlington, MA, USA) at 2 weeks after weaning. ${ }^{9}$ Blood glucose levels were measured by a blood glucose and ketone monitoring system (98814-65, Abbott, Chicago, IL, USA) 4 days after injection to confirm whether T1D was completely induced. Mice with blood glucose levels higher than $1.67 \mathrm{mM}$ (300 mg/dL) were considered to have induced T1D (Fig. 1). ${ }^{16}$
Three weeks after the blood glucose check, the body weights of control, AKITA, and STZ-injected mice were measured. A total of 150 mice [wild-type C57BL/6J ( $n=50)$, AKITA ( $n=50)$, and STZ-injected mice $(\mathrm{n}=50)]$ were used in this study.

We did not use homozygous AKITA mice because they rarely survive beyond 12 weeks of age without insulin treatment. ${ }^{17}$ Although homozygous AKITA mice may be more relevant to human T1D, the homozygous AKITA mouse was not ideal for evaluating ovarian functional endpoints due to their early mortality.

\section{Genotyping}

Genomic DNA (gDNA) was extracted from tail tips and used for PCR. We followed Jackson Laboratory's protocol for genotyping. A set of primers (forward: 5'-TGCTGATGCCCTGGC CTGCT-3', reverse: 5'-TGGTCCCACATATTGCACATG-3') was used to amplify the gDNA. PCR was performed according to the manufacturer's instructions (K0171, Thermo Fisher Scientific, Waltham, MA, USA) and was followed by digestion with the restriction enzyme Fnu4HI (R0178, New England Biolabs, Ipswich, MA, USA) at $37^{\circ} \mathrm{C}$ overnight. PCR products of 140 base pairs (bp) and $280 \mathrm{bp}$ indicated the presence of the wildtype allele and AKITA mutant allele, respectively. ${ }^{10}$

\section{Ovarian morphology and immunoblot analysis}

Ovary size was compared in control, AKITA, and STZ-injected mice by gross observation. Follicle number and growth was assessed based on immunoblot analysis of anti-Mullerian hormone (AMH), follicle-stimulating hormone receptor (FSHR), and luteinizing hormone/chorionic gonadotropin receptor (LHCGR), which are exclusively expressed in granulosa cells and theca cells of ovarian follicles. Ovarian tissues from each group were homogenized according to a previous report, ${ }^{18}$ and total protein was collected for immunoblot analysis. Primary antibodies were rabbit anti-AMH (PA5-35851, Thermo Fisher Scientific, Waltham, MA, USA; 1:2000), anti-FSHR (PA550963, Thermo Fisher Scientific, Waltham, MA, USA; 1:2000), and anti-LHCGR (SC-25828, Santa Cruz Biotechnologies, Dallas, TX, USA; 1:2000), and mouse anti-alpha-tubulin (T9026, MilliporeSigma, Burlington, MA, USA; 1:5000). Secondary antibodies were horseradish peroxidase (HRP)-conjugated goat anti-rabbit or anti-mouse (65-6120, 65-6520, Thermo Fisher Scientific, Waltham, MA, USA; 1:5000). A sample of $20 \mu \mathrm{g}$ of total protein was separated by SDS-PAGE (NP0335 BOX, Thermo Fisher Scientific, Waltham, MA, USA) at $150 \mathrm{~V}$ for $2 \mathrm{hr}$ and then transferred to a polyvinylidene difluoride membrane (IB23002, Thermo Fisher Scientific, Waltham, MA, USA). The membranes were then blocked at room temperature for $1 \mathrm{hr}$ and incubated with primary antibodies at $4^{\circ} \mathrm{C}$ overnight. Membranes were then washed three times and incubated with secondary antibodies at room temperature for $1 \mathrm{hr}$. The blots were developed with Luminata Crescendo Western HRP substrate (WBLU0500, MilliporeSigma, Burlington, MA, USA). Images 
were captured by Azure C500 (Azure C500, Azure Biosystems, Dublin, CA, USA).

\section{Histological and immunohistochemical analysis of ovarian tissue}

Three weeks after a single STZ injection, control, AKITA, and STZ-injected mice underwent gonadotropin hyperstimulation and the ovaries were collected. The mean number of corpora lutea (CLs) was counted on the surface of whole ovaries. The ovaries were then fixed with $4 \%$ paraformaldehyde (PFA) and embedded in paraffin wax, then serially sectioned at 5 $\mu \mathrm{m}$ thickness. Ovarian tissues were stained with hematoxylin and eosin (H\&E, Merck, Kenilworth, NJ, USA) for histological analysis. For immunofluorescence, the paraffin sections were incubated with anti-AMH (sc-6886, Santa Cruz Biotechnology, Dallas, TX, USA; 1:100), anti-estrogen receptor-beta (ER-beta, sc-6821, Santa Cruz Biotechnology, Dallas, TX, USA; 1:50), Ki67 (652401, BioLegend, San Diego, CA, USA; 1:50), and antiMSY2 (a gift from Dr. Richard Schultz, University of Pennsylvania, 1:4000) antibodies. Alexa Fluor 488 donkey anti-rabbit IgG $(\mathrm{H}+\mathrm{L})$ was used as the secondary antibody. ${ }^{19}$ To counterstain the nucleus, the slides were mounted with DAPI [2-(4-amidinophenyl)-1H-indole-6-carboxamidine, Vector Laboratories, Burlingame, CA, USA] mounting solution.

Hormone stimulation and evaluation of oocyte quantity Eight-week-old female mice were hormonally primed by intraperitoneal injection of 5 IU equine chorionic gonadotropin (367222, equine chorionic gonadotropin, MilliporeSigma, Burlington, MA, USA) and then 5 IU human chorionic gonadotropin (hCG) (CG10-1VL, MilliporeSigma, Burlington, MA, USA) $48 \mathrm{hr}$ later. At $16 \mathrm{hr}$ after hCG administration, metaphase II oocytes were collected from the oviduct to assess oocyte quality. The number and stage of oocytes retrieved after superovulation was determined to evaluate in vivo folliculogenesis and oocyte maturation in control, AKITA, and STZ-injected mice.

\section{Serum hormone ELISA}

After hormonal stimulation to induce superovulation, whole blood was collected and the serum separated by centrifugation at 3000 RPM for $30 \mathrm{~min}$. ELISAs for insulin (EZRMI-13K, Calbiochem, San Diego, CA, USA), AMH (MBS2507173, MyBioSource, San Diego, CA, USA), 17 beta-estradiol (E2, ES180S, Calbiochem, San Diego, CA, USA), testosterone (T, TE187S, Calbiochem, San Diego, CA, USA), and progesterone (P4, PG129S, Calbiochem, San Diego, CA, USA) were performed to compare levels of each hormone between control, AKITA, and STZ-injected mice.

\section{Immunofluorescence of oocyte structures after superovulation}

Meiotic spindle formation and the distribution of cortical granules were investigated to determine oocyte quality. To detect the intra-oocyte meiotic spindle, chromosomal alignment, and cortical granules, metaphase II oocytes retrieved from control, AKITA, and STZ-injected mice were denuded by treating with $85 \mathrm{IU} / \mathrm{mL}$ hyaluronidase (H4272, MilliporeSigma, Burlington, MA, USA) and then immediately fixed with $4 \%$ PFA (15710, Electron Microscopy Sciences, Hatfield, PA, USA). Following fixation, the oocytes were permeabilized by treating with $0.1 \%$ Triton X-100 (X100, MilliporeSigma, Burlington, MA, USA) in phosphate-buffered saline (14190-250, Thermo Fisher Scientific, Waltham, MA, USA) and then blocked with 3\% BSA in PBS (A0281, Thermo Fisher Scientific, Waltham, MA, USA) at room temperature for $1 \mathrm{hr}$. Oocytes were then incubated with rabbit anti- $\alpha$-tubulin antibody (5063S, Cell Signaling Technology, Danvers, MA, USA; 1:100) at $4^{\circ} \mathrm{C}$ overnight to detect the meiotic spindle. To localize cortical granules, oocytes were incubated with rhodamine-conjugated Lens Culinaris Agglutinin (RL-1042, LCA, Vector Laboratories, Burlingame, CA, USA; 1:100) at room temperature for $1 \mathrm{hr}$. After staining, the oocytes were mounted with 4,6-diamidino2-phenylindole mounting solution (H-1200, Vector Laboratories, Burlingame, CA, USA) and then examined under a TCS SP5 confocal microscope (SP5, Leica, Wetzlar, Germany). Oocytes with barrel-shaped bipolar spindles, well-organized microtubule fibers, and tightly aligned chromosomes on the metaphase plate were scored as normal. All other configurations were considered abnormal. ${ }^{20,21}$

\section{Measurement of mitochondrial function $(\Delta \psi \mathrm{m})$ and mtDNA}

Following oocyte denudation with $0.1 \%$ bovine testes hyaluronidase (MilliporeSigma, Burlington, MA, USA), metaphase II oocytes were stained with JC-1 dye (T3168, Thermo Fisher Scientific, Waltham, MA, USA) at $37^{\circ} \mathrm{C}$ for $30 \mathrm{~min}$. Oocytes were then transferred into a mineral oil-covered Leibovitz-15 medium (11415-114, Thermo Fisher Scientific, Waltham, MA, USA) and green/red fluorescence was captured by confocal microscopy to evaluate high (green) and low (red) mitochondrial membrane potential of the metaphase II oocytes, as described in previous studies. ${ }^{22,23}$

Mitochondrial DNA from individual metaphase II oocytes was isolated, and the copy number was determined as described previously by Dr. Robker's group ${ }^{24}$ Briefly, oocytes isolated from wild-type control, AKITA, and STZ-injected mice were washed with $0.1 \%$ polyvinylpyrrolidone (PVP) (P5288, MilliporeSigma, Burlington, MA, USA) in PBS and then transferred into $5 \mu \mathrm{L}$ of $0.1 \%$ PVP in PBS. Carrier RNA $(1 \mu \mathrm{g})$ was then added into each sample, and the DNA was isolated using the QIAamp DNA Micro Kit (56304, Qiagen, Venlo, Netherlands) according to the manufacturer's instructions. DNA copy number (mtDNA) was eluted with $50 \mu \mathrm{L}$ of distilled water and used for quantitative PCR. A standard curve for absolute quantification of copy number was generated based on $10^{1}$ to $10^{7}$ copies, kindly provided by Dr. Robker's group. The primers used for 
PCR were 5'-CGTTAGGTCAAGGTGTAGCC-3' and 5'-CCA GACACACTTTCCAGTATG-3'. All experiments were carried out at least three times. The mtDNA copy number in individual oocytes was determined by comparing the calculated cycle threshold value against the corresponding standard curve as described in previous studies. ${ }^{25,26}$

\section{Live cell fluorescence measurement of calcium}

Intracellular calcium $\left[\left(\mathrm{Ca}^{2+}\right) \mathrm{i}\right]$ measurement in activated oocytes was performed as previously described. ${ }^{25,27}\left(\mathrm{Ca}^{2+}\right)_{\mathrm{i}}$ was measured using the $\mathrm{Ca}^{2+}$ sensitive dye Fura-2-acetoxymethyl ester (F1221, Fura 2-AM, Thermo Fisher Scientific, Waltham, MA, USA). Oocytes were monitored simultaneously using an inverted Nikon Eclipse Ti microscope (Eclipse Ti, Nikon, Shinagawa, Tokyo, Japan) outfitted with fluorescence measurement capabilities. Ionomycin stock (I9657, MilliporeSigma, Burlington, MA, USA) solution was introduced into the imaging drop 1 min after the start of monitoring. The Fura 2-AM dye was stimulated between $340 \mathrm{~nm}$ and $380 \mathrm{~nm}$ by a filter wheel (Lambda 10-3, Sutter Instrument, Novato, CA, USA), and fluorescence was captured every $4 \mathrm{sec}$. Emitted light was collected by a CoolSnapES2 CCD camera (Photometrics, Tucson, AZ, USA). Fluorescence ratios were analyzed by ImageJ (Bethesda, MD, USA).

\section{In vitro fertilization}

In vitro fertilization (IVF) was carried out using cumulus-oocyte complexes (COCs) containing metaphase II oocytes from 8-week-old wild-type control, AKITA, and STZ-injected mice. Epididymal spermatozoa were collected from wild-type mice and capacitated to ensure penetration and fertilization of the metaphase II oocytes. For capacitation, the collected spermatozoa were incubated in $0.4 \%$ bovine serum albumin containing EmbryoMax ${ }^{\circledR}$ human tubal fluid (HTF) (MR-070-D, MilliporeSigma, Burlington, MA, USA) at $37^{\circ} \mathrm{C}, 100 \%$ humidity, and $5 \% \mathrm{CO}_{2}$ atmosphere. COCs were isolated from the ampulla region of the oviduct and pre-incubated in HTF medium. Following capacitation, approximately 1 to $2 \times 10^{6}$ fully capacitated motile spermatozoa were incubated with COCs for $4 \mathrm{hr}$. The fertilized oocytes were then isolated and transferred into a potassium simplex optimized medium (EmbryoMax ${ }^{\circledR}$ KSOM Medium, MR-121-D, MilliporeSigma, Burlington, MA, USA) for 5 days at $37^{\circ} \mathrm{C}$ in $5 \% \mathrm{CO}_{2}$. Cleavage (\% of two-cell embryos/ oocytes fertilized) and blastocyst formation rate (\% of blastocyst/oocytes fertilized) were then measured as markers of embryonic developmental competence. ${ }^{27}$

\section{Blastomere TUNEL staining}

Five days after IVF, blastocysts from wild-type control, AKITA, and STZ-injected mice were immediately fixed with $4 \%$ PFA and then washed three times with $0.1 \%$ PVP. The blastocysts were then incubated with $0.1 \%$ Triton X-100 in PBS at room temperature for $30 \mathrm{~min}$. To visualize blastomere apoptotic status, blastocysts were treated with an incubation buffer containing equilibration buffer, nucleotide mix, and rTdT enzyme (G3250, Promega, Madison, WI, USA) at $37^{\circ} \mathrm{C}$ for $1 \mathrm{hr}$. Blastocysts were mounted with DAPI-containing mounting solution. The apoptotic and total blastomere numbers were measured by Z-stack confocal microscopy. ${ }^{28}$

\section{Statistical analysis}

Data were analyzed by either Chi-square test (oocyte maturation, spindle normality, and embryonic development after IVF) or one-way ANOVA (body weight, blood glucose, hormone measurements, number of oocytes retrieved after superovulation, number of CL, and apoptotic and total blastomeres) using SPSS ver. 12.0 (SPSS Inc., Chicago, IL, USA) or GraphPad Prism 5.0 (GraphPad Software, San Diego, CA, USA). Tukey's multiple comparison was used as a post-hoc test. Values were considered statistically significantly different at $p<$ 0.05 .

\section{RESULTS}

\section{Characteristics of T1D mouse models}

Fig. 1A shows the PCR-based confirmation of ins 2 mutant heterozygosity in the AKITA mouse model of T1D (presence of both 140 bp and 280 bp Fnu4HI digestion products). STZinjected mice had elevated blood glucose and significantly lower body weight compared with wild-type control mice. While the heterozygous AKITA mice also showed higher blood glucose levels, body weights were comparable between AKITA and wild-type control mice (Fig. $1 \mathrm{~B}$ and C, $p<0.05$ ).

\section{The HPO axis is impaired by STZ treatment in mice}

To investigate the effect of diabetes on female reproductive tissues, ovaries from 8-week-old control, AKITA, and STZ-injected mice were harvested (Fig. 1D). Ovary size was not significantly different in control and AKITA mice, whereas ovaries from STZ-injected mice were substantially smaller 3 weeks after injection. To assess the function of granulosa and theca cells in ovarian follicles in the three groups of mice, we examined protein expression levels of AMH, FSHR, and LHCGR. Consistent with the smaller ovary size, STZ-injected ovaries had relatively low expression levels of $\mathrm{AMH}, \mathrm{FSHR}$, and LHCGR protein (Fig. 1E, left; control, middle; AKITA, and right; STZ-injected). Immunofluorescence showed uneven expression of AMH in antral follicles in the ovaries of AKITA mice, while it was uniformly expressed in granulosa cells of growing follicles (primary, secondary, and multilayered secondary follicles). ${ }^{16}$ Furthermore, the expression of ER, a granulosa cell marker, was barely detectable in the granulosa cells of antral follicles from AKITA and STZ-injected mice, while high expression levels of Ki-67 were detected in proliferating granulosa cells in all three groups (Fig. 1F). This suggests that hyper- 


\section{A}
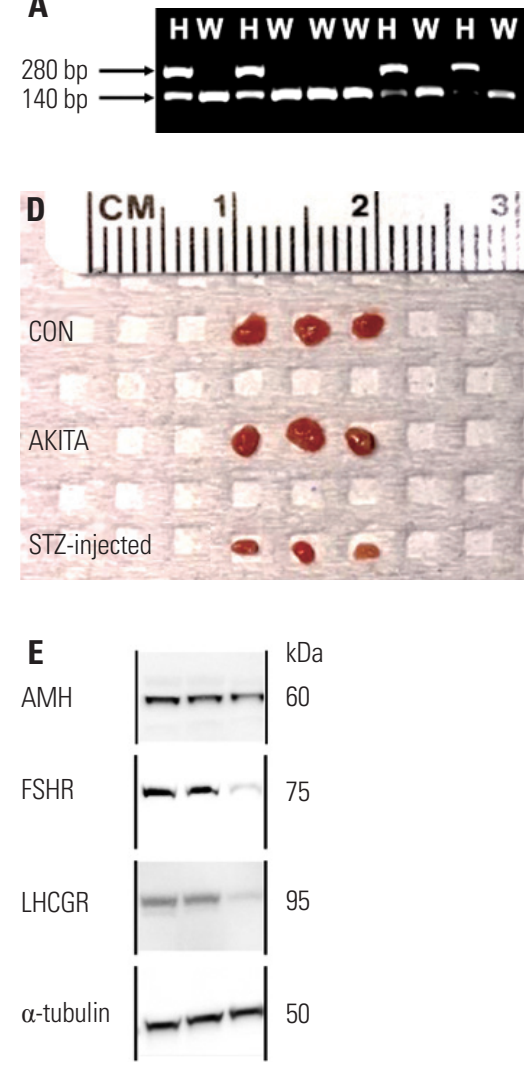

B

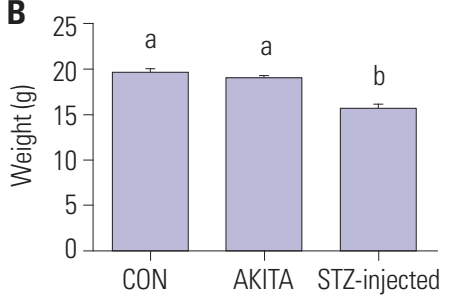

$\mathbf{F}$
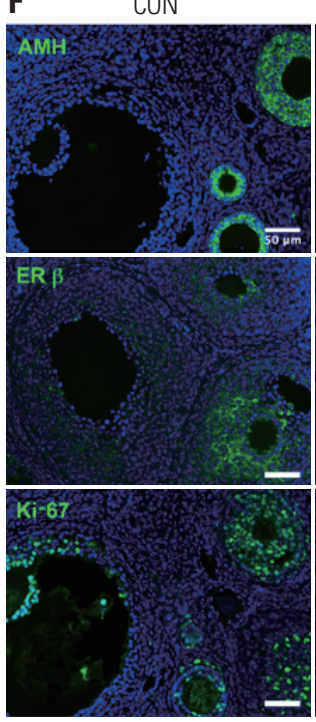

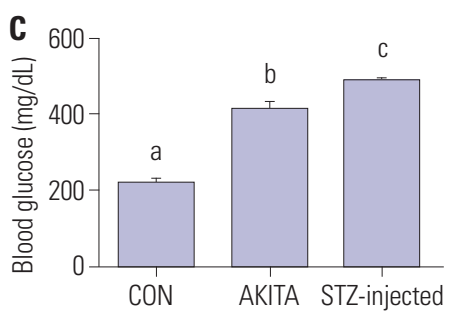

AKITA
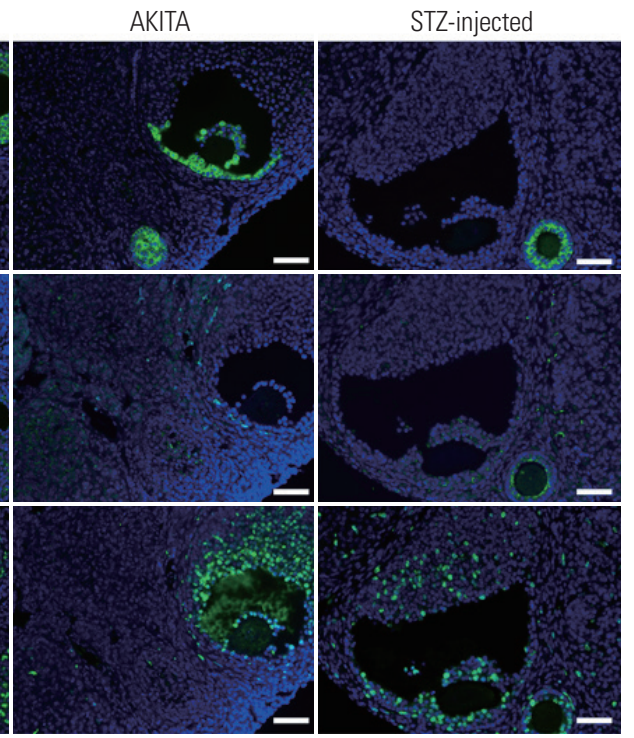

Fig. 1. Characteristics of animals used in this study and impairment of the hypothalamus-pituitary-ovary axis in streptozotocin-induced type I diabetes mellitus. (A) PCR and restriction enzyme digestion were carried out to confirm the presence of the ins2 gene mutation. Control and AKITA mice showed different PCR products after digestion. (B) The streptozotocin (STZ)-injected mice had a lower body weight and (C) higher blood glucose levels, compared to control mice $(n=15)$. Different letters indicate a statistically significant difference $(p<0.05)$. (D) Ovary size was dramatically reduced 3 weeks after STZ injection, while AKITA mice did not show any reduction in ovary size $(\mathrm{n}=6)$. (E) In STZ-injected mice, the expression of FSHR and luteinizing hormone/chorionic gonadotropin receptor (LHCGR) was substantially diminished compared with control and AKITA mice. (F) The expression of anti-Mullerian hormone (AMH), anti-estrogen receptor-beta (ER-beta), and Ki-67 in the ovaries from control, AKITA, and STZ-injected mice (50 $\mu \mathrm{m}$, $\times 200)$. The expression levels of these proteins, which are correlated with folliculogenesis and proliferation, were reduced in STZ-injected mice. Different letters ( $a$, b, c) indicate a statistical significant difference. H, heterozygous; W, wild type; CON, control; FSHR, follicle stimulating hormone receptor.

glycemia affects the expression patterns and levels of ovarian proteins in the diabetes mouse models.

\section{Ovarian histology and the quantity and quality of oocytes following ovarian hyperstimulation are affected by T1D in mice}

The ovaries in all three groups of mice contained growing follicles at all stages and antral follicles. All mice responded to stimulation with exogenous gonadotropin (Fig. 2A). Although STZ-injected mice had smaller ovaries compared with control and AKITA mice, follicle growth was stimulated by exogenous gonadotropins. Ovarian histology revealed antral follicle stages (black arrows) and CLs (yellow arrows), although the ovaries of STZ-injected mice contained CL with "trapped" oocytes (red arrow), suggesting impairment of the ovulation process (Fig. 2A). Interestingly, Fig. 2B shows a normal structure of ovary while, we observed abnormal morphology of COCs, such as pyknotic nuclei in granulosa cells in AKITA mice (inset, Fig. 2B) and disconnection of oocytes and granulosa cells in both AKITA and STZ-injected mice (Fig. 2B). To con- firm that oocytes were "trapped" in CLs in STZ-injected mice, we conducted an immunofluorescence assay with MSY2, an oocyte-specific marker; a positive green signal indicated oocytes of growing follicles. Oocytes trapped in the CL, even after exogenous hCG stimulation, appeared only in STZ-injected mice. Trapped and ruptured oocytes showed diffuse MSY2 signals, in contrast to strong signals in growing follicles (Fig. 2C). In addition, STZ-injected mice produced significantly fewer metaphase II oocytes following hormonal stimulation compared with control mice (Fig. 2D), with significantly fewer CLs in STZ-injected mice (Fig. 2E). Oocyte maturation to the metaphase II stage after hCG treatment was less frequent in oocytes collected from AKITA and STZ-injected mice compared to control mice (Fig. 2F).

\section{T1D mice show variations in hormonal response to exogenous hormone stimulation}

As expected, both AKITA and STZ-injected mouse models of T1D showed dramatically decreased serum insulin levels at 8 weeks of age (Fig. 3A, $p<0.05$ ). Serum AMH, 17-beta-estradiol 

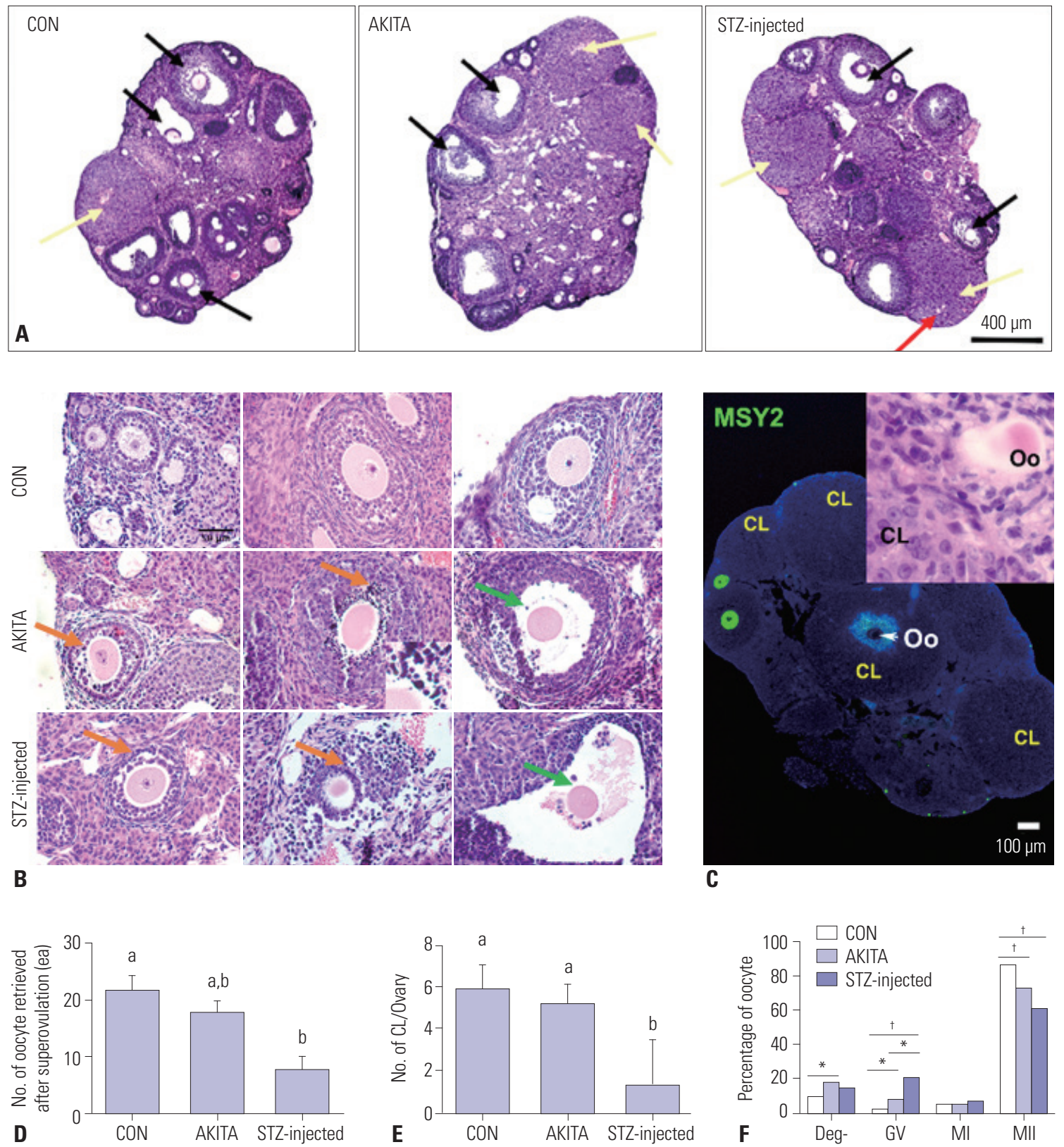

Fig. 2. Histological observation of oocyte quantity and quality after superovulation. (A) All stages of ovarian follicles and the corpus luteum (CL) were seen in control, AKITA, and streptozotocin (STZ)-injected mice. Black arrows indicate antral follicles, yellow arrows show CL, and the red arrow indicates trapped oocytes in CLs. CLs and oocytes are marked $(400 \mu \mathrm{m}, \times 200)$. (B) Both diabetic mice models showed pyknotic nuclei of granulosa cells (orange arrows) and impairment of oocyte-granulosa cell connection (green arrows), while the growing follicles in control mice showed normal morphology $(50 \mu \mathrm{m}, \times 200)$. (C) Ovarian tissue stained with oocyte-specific marker (MSY2) to demonstrate trapped oocytes in CL (100 $\mu \mathrm{m}, \times 200)$. An inset indicates trapped oocytes in CL. Orange arrows and green signals indicate the oocytes of different follicle stages (B, C). The white arrow and letter ' $0 o^{\prime}$ indicate trapped oocytes in CLs, which are also shown in $(A)$. (D and E) The numbers of oocytes and CLs in the ovary after superovulation were significantly lower in STZ-injected mice. Different letters $(\mathrm{a}, \mathrm{b})$ indicate a statistically significant difference $(p<0.05, \mathrm{n}=6)$. (F) Oocyte maturation in diabetic mice was also impaired, compared with control. * and ${ }^{\dagger}$ respectively indicate $p<0.05$ and $p<0.001(\mathrm{n}=80)$. CON, control; 00, 00cyte; Deg-, degenerated; GV, germinal vesicle; MI, germnial vesicle breakdown; MII, metaphase II.

(E2), and testosterone ( $\mathrm{T}$ ) levels following exogenous hormone stimulation and superovulation were comparable between control mice and the T1D mice (Figs. 3B-D). Serum progesterone levels were lower in STZ-injected mice than in control mice (Fig. 3E). This indicated that growing follicles in diabetic mice could be stimulated by exogenous hormone priming.

TID mice have abnormal meiotic spindle formation in metaphase II oocytes

Fig. 4A shows the distribution of cortical granules (red) and DNA (blue) in metaphase II oocytes collected from control, 

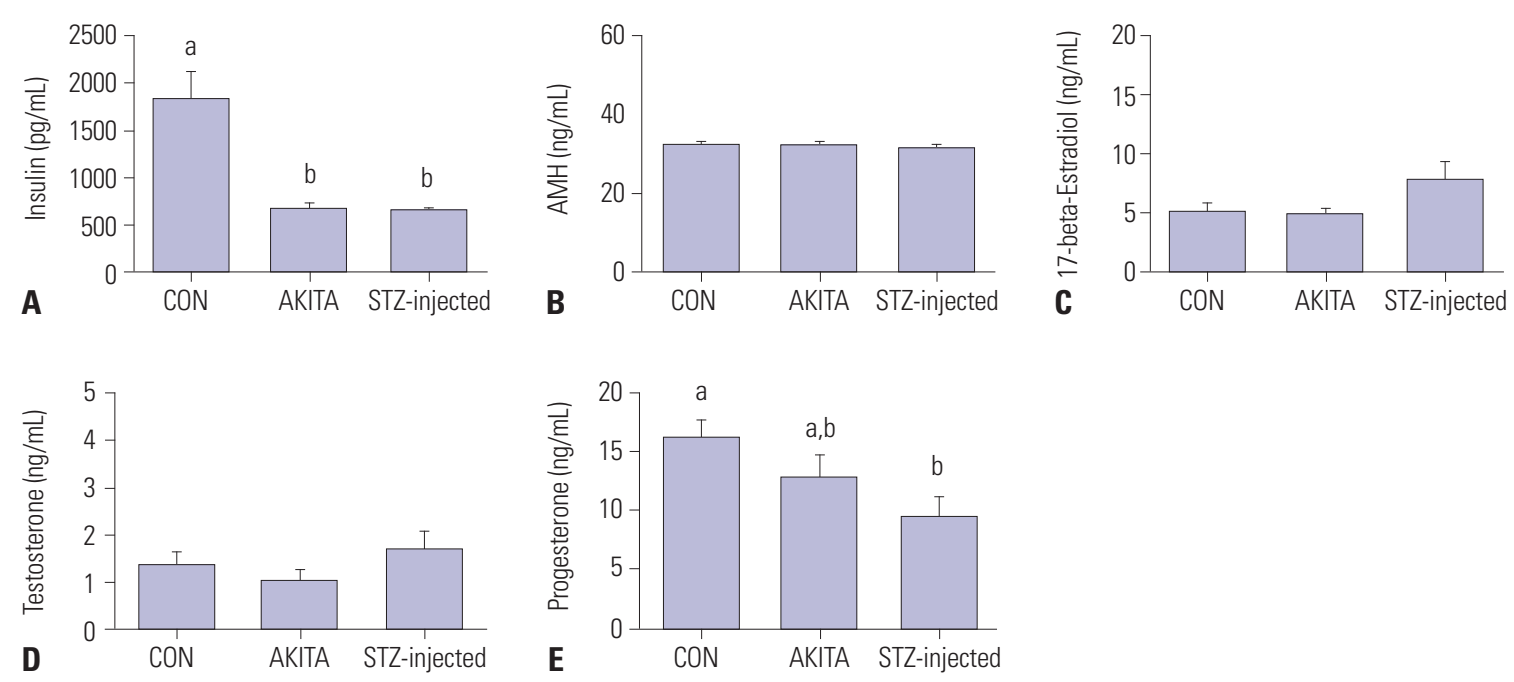

Fig. 3. (A-E) Hormone profiles after superovulation. (A-E) Insulin, anti-Mullerian hormone (AMH), 17-beta-estradiol, testosterone, and progesterone were measured. Diabetic mice had lower insulin and progesterone levels, whereas 17-beta-estradiol and testosterone levels were comparable between control and diabetic mice. Different letters $(a, b)$ indicate a statistically significant difference $(p<0.05)$. STZ, streptozotocin; CON, control.

AKITA, and STZ-injected mice. Representative images of oocytes with localization of the meiotic spindle with tubulin staining (green) and DNA with DAPI (blue) are shown in Fig. 4B. Abnormal oocyte spindle formation was observed more frequently in oocytes from the T1D mouse models compared to oocytes from control mice (Fig. 4C).

\section{T1D affects mitochondrial membrane potential and DNA copy number in metaphase II oocytes}

Fig. 5A shows JC-1 staining of the mitochondrial membrane potentials associated with oocyte quality. Red and green dots, respectively, indicate high and low mitochondrial membrane potentials in the metaphase II oocytes. Metaphase II oocytes from both T1D mouse models showed a significantly lower mitochondrial membrane potential compared with metaphase II oocytes from control mice (Fig. 5B). However, mtDNA copy number was comparable across all three mouse groups, as shown in Fig. 5C. Intracellular calcium release in activated oocytes was also comparable between control and T1D mice (Figs. 5D and E)

\section{T1D mice have reduced embryonic developmental competence of metaphase II oocytes}

In vitro fertilized oocytes from both AKITA and STZ-injected mice showed significantly impaired developmental competencies. Compared to control mice, fewer fertilized eggs obtained from T1D mice underwent cleavage at $24 \mathrm{hr}$ (Fig. 6A) and reached the blastocyst stage by $120 \mathrm{hr}$ (Fig. 6B). In addition, the number of blastomeres per blastocyst was significantly lower in T1D mice compared to controls (Fig. 6C), and fertilized oocytes retrieved from T1D mice also contained a higher number of apoptotic blastomeres than fertilized oocytes from control mice (Fig. 6D). Fig. 6E shows a representative image of terminal deoxynucleotidyl transferase dUTP nick end-labeling (TUNEL) and DAPI staining of a blastocyst following IVF.

\section{DISCUSSION}

In this study, we compared various markers of ovarian function, from ovarian health and oocyte quality to embryo developmental competence, between wild-type mice and two different mouse models of T1D (AKITA and STZ-induced). We found that, compared to wild-type control and AKITA mice, STZ-induced T1D mice showed more severe impairment of LHCGR signaling-associated events, including luteinization, oocyte maturation, and serum progesterone production. Metaphase II oocytes from both T1D mouse models showed impaired spindle formation, lower mitochondrial membrane potential, and lower developmental competence after IVF compared with oocytes from wild-type mice.

Though the severity of T1D in various animal models might differ, only a few studies have compared AKITA and STZ-injected mouse models in relation to function in the ovary.,29-31 We found that mice injected with STZ showed a more severe hyperglycemic condition and lower body weight than the AKITA mice, which may underlie the observed differences in reproductive endpoints between the two models.

Previous studies have described the development of hypogonadism as a complication of T1D. ${ }^{29-31}$ According to our data, AKITA mice did not show evidence of hypogonadism compared to wild-type controls, despite the presence of hypoinsulinemia and hyperglycemia. Schoeller, et al. ${ }^{30,31}$ similarly described no difference in testis size between male heterozygous AKITA and wild-type control mice. In contrast, we observed that mice with T1D induced by STZ showed markedly reduced ovary size compared to controls, with lower levels of FSHR and 

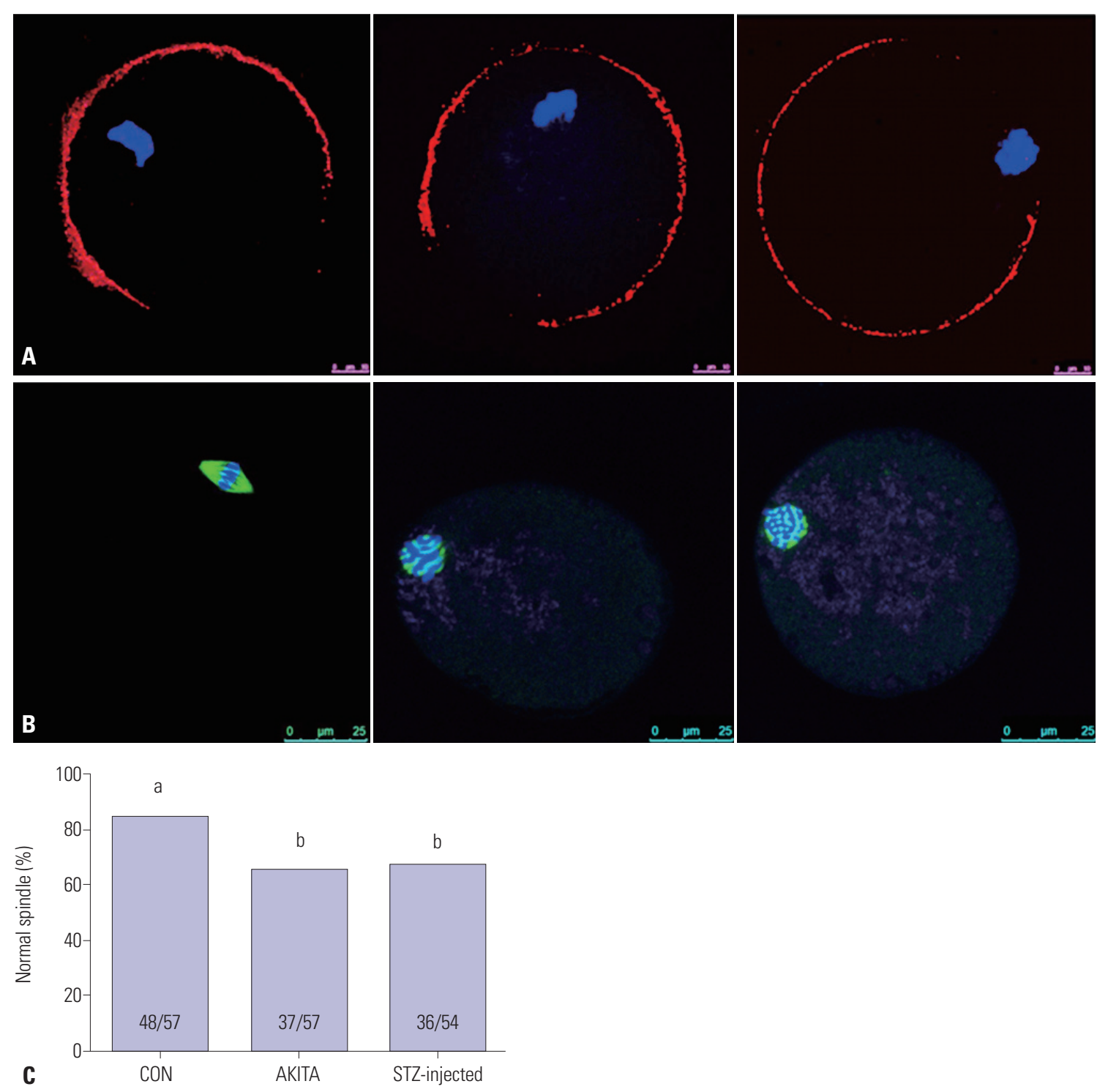

Fig. 4. Microorganelles in mature oocytes and meiotic spindle formation. (A) Cortical granule (red) distribution in mature oocytes derived from wildtype, AKITA, and streptozotocin (STZ)-injected mice $(n=20)(10 \mu \mathrm{m})$. Blue indicates DNA of chromsome which was stained by 2-(4-amidinophenyl)-1H -indole-6-carboxamidine (DAPI). (B) Meiotic spindle (green) and DNA (blue) localized in mature oocytes. (C) The proportion of normal meiotic spindles. (C) Different letters $(a, b)$ indicate statistically significant differences $(p<0.05)$. CON, control.

LHCGR expression. The previous study by Schoeller, et al. ${ }^{30,31}$ found that homozygous AKITA male mice, which have a more severe phenotype with lower serum insulin compared to heterozygous AKITA male mice, also showed more severe hypogonadism and reductions in serum LH. This suggests that the severity of T1D may determine the impact of the disease on the HPO axis, particularly LHCGR signaling, in diabetic mice.

Interestingly, the function of the HPO axis could be partially restored by exogenous gonadotropin in STZ-injected mice. The ovaries from both T1D mouse models contained all stages of growing, pre-ovulatory follicles and CLs, as well as normal E2 and T levels, comparable to wild-type controls. Johnson and Sidman ${ }^{32}$ showed normal sensitivity of target organs to gonadotropin and sex hormones in female T1D mice. Schoeller, et al. ${ }^{30}$ also reported no significant difference in serum FSH lev- els between wild-type control and AKITA mice. Taken together with our findings, this suggests that the FSH responsiveness of growing follicles is preserved in T1D mice. Conversely, Schoeller, et al. ${ }^{30,31}$ found that serum LH levels were lower in both heterozygous and homozygous AKITA mice, and brainspecific knockout of the insulin receptor impaired spermatogenesis by reducing circulating $\mathrm{LH}$. We found that STZ-induced T1D mice had a markedly decreased number of ovulated oocytes and CLs after hormonal stimulation, a reduced level of serum progesterone, and impaired oocyte maturation. Based on these findings, we conclude that poorly controlled, severe T1D might lead to impairment of later stages of folliculogenesis and oocyte maturation, which are strongly associated with LHCGR signaling, through a decrease in circulating LH and LHCGR expression, rather than changes in FSH. 

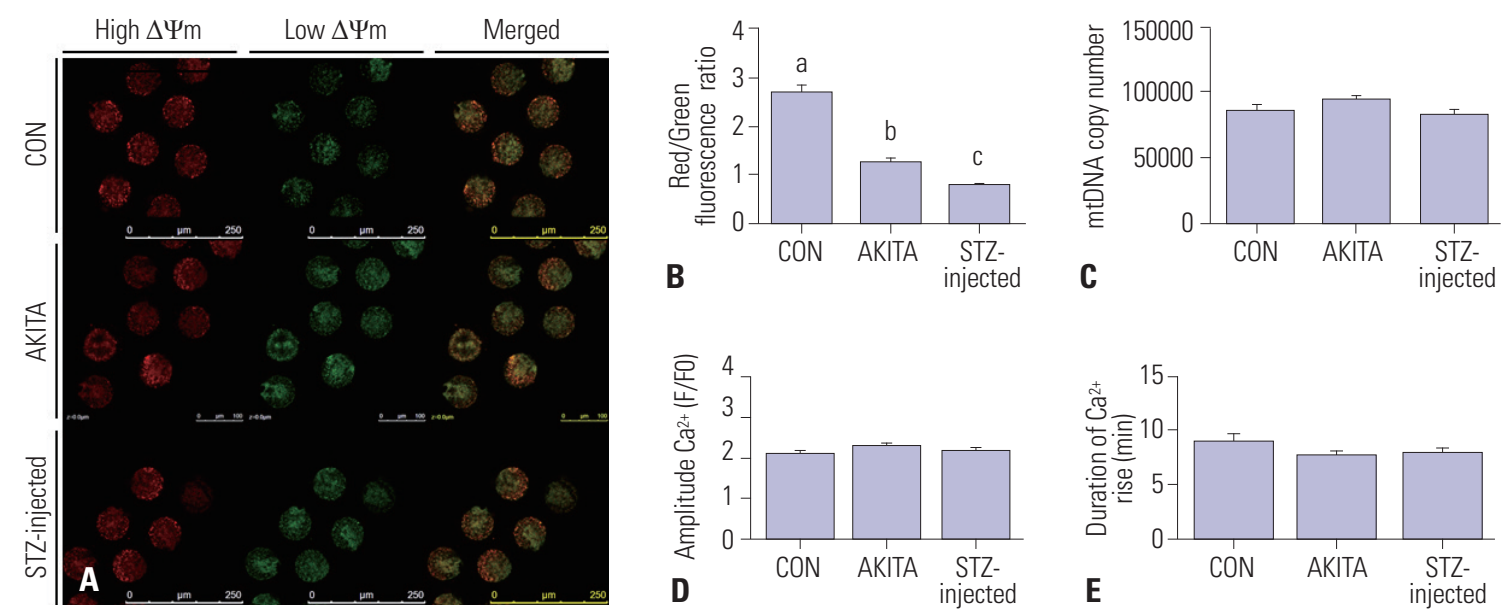

Fig. 5. Mitochondrial characteristics and exocytosis of calcium from mature oocytes. (A) A representative image of JC-1 staining of mature oocytes. Red and green indicate high and low mitochondrial membrane potential, respectively. Magnification was $\times 200$. (B) The ratios of red/green fluorescence intensity were measured, and both diabetic mouse models had significantly lower mitochondrial membrane potential compared with controls. Different letters $(\mathrm{a}, \mathrm{b}, \mathrm{c})$ indicate a statistically significant difference $(p<0.05, \mathrm{n}=14)$. (C) The quantity of mtDNA was similar between control and diabetic mouse groups $(n=18)$. The exocytosis of calcium ( $D$ and $E$ ) was also comparable in oocytes from control and diabetic mice $(n=15)$. STZ, streptozotocin; CON, control.

Studies documenting oocyte quality in AKITA and STZinjected T1D mice have been conducted by the Moley group., ${ }^{9,26}$, ${ }^{30,31,33,34}$ In 2005, they found that T1D mice exhibit delayed germinal vesicle breakdown after hCG treatment and reduced connexin-43 expression in cumulus-enclosed oocytes. ${ }^{6}$ Oocytes from female T1D mice showed evidence of altered mitochondrial ultrastructure, mtDNA copy number, and adenosine triphosphate (ATP) and tricarboxylic acid (TCA) cycle metabolites due to mitochondrial metabolic dysfunction. ${ }^{9,33,34}$ In addition, female T1D mice had a greater frequency of oocyte spindle or chromosomal misalignment leading to aneuploidy. Finally, they showed that TID disrupted crosstalk between the oocyte and cumulus cells by inducing mitochondrial impairment in cumulus cells. ${ }^{9}$ Similar to their studies, we found significantly increased spindle abnormalities in both AKITA and STZ-injected TID mice; however, there was no difference in cortical granule distribution in T1D mice compared to control, which had not been investigated previously in metaphase II oocytes. Some research groups previously demonstrated that T1D mice exhibit abnormal, dynamic changes in the ER, granulosa cell apoptosis, abnormal energy metabolism, and mitochondrial dysfunction in oocytes. ${ }^{6,9,33}$ Recent papers have described that cortical granules can change the structure of zona pelluci$\mathrm{da}^{35,36}$ and can cause low fertility in diabetic mice. In this regard, we analyzed cortical granules in oocytes retrieved from both healthy and diabetic mice. We found a reduced mitochondrial membrane potential in both T1D mouse models compared to controls, but no change in mitochondrial DNA copy number. Wai, et al..$^{37}$ demonstrated that oocytes with as few as 4000 copies of mtDNA can be fertilized and develop to the blastocyst stage, and that only 40000-50000 copies of mtDNA are required to permit fetal development. As there was no significant difference in mtDNA copy number in metaphase II oocytes between the T1D and wild-type control groups, it is likely that oocytes from either TID model possess the potential to develop into a fetus.

Zhang, et al. ${ }^{38}$ demonstrated that T1D mice develop abnormal dynamic changes in the ER during oocyte maturation and early embryo development. We expected that the T1D-related abnormal distribution of ER would have a deleterious impact on calcium physiology during activation of the metaphase II oocyte. However, the amplitude and duration of calcium fluctuations after ionomycin-derived egg activation were comparable between control and both T1D mouse models. Thus, ER function, not ER distribution, appears to have been preserved in our T1D mouse models.

Finally, we found that T1D decreases pre-implantation embryonic development and promotes blastomere apoptosis $120 \mathrm{hr}$ after IVF. Alterations in microorganelles, function, physiology, and ATP levels in metaphase II oocytes may mediate the detrimental effects of T1D on developmental competence after IVF. Moley, et al. ${ }^{39}$ found that pre-implantation embryos from diabetic mice exhibit a shift in glucose transporter 1-3 (GLUT 1-3) utilization. Additionally, Chi, et al. ${ }^{40} \mathrm{de}-$ scribed a decline in GLUT 1 utilization, which may promote blastomere apoptosis at the blastocyst stage. Thus, our observation of an increase in total and apoptotic blastomeres in T1D mice may be related to impairment of glucose uptake and metabolism in pre-implantation embryos.

Collectively, we found that the STZ-induced mouse model of T1D, which developed more severe hyperglycemia compared to the AKITA mouse model, showed greater impairment of LHCGR signaling-related events, including ovulation, oocyte maturation, and luteinization, and pre-implantation embryonic development. Both T1D mouse models showed significantly decreased oocyte and pre-implantation embryo 

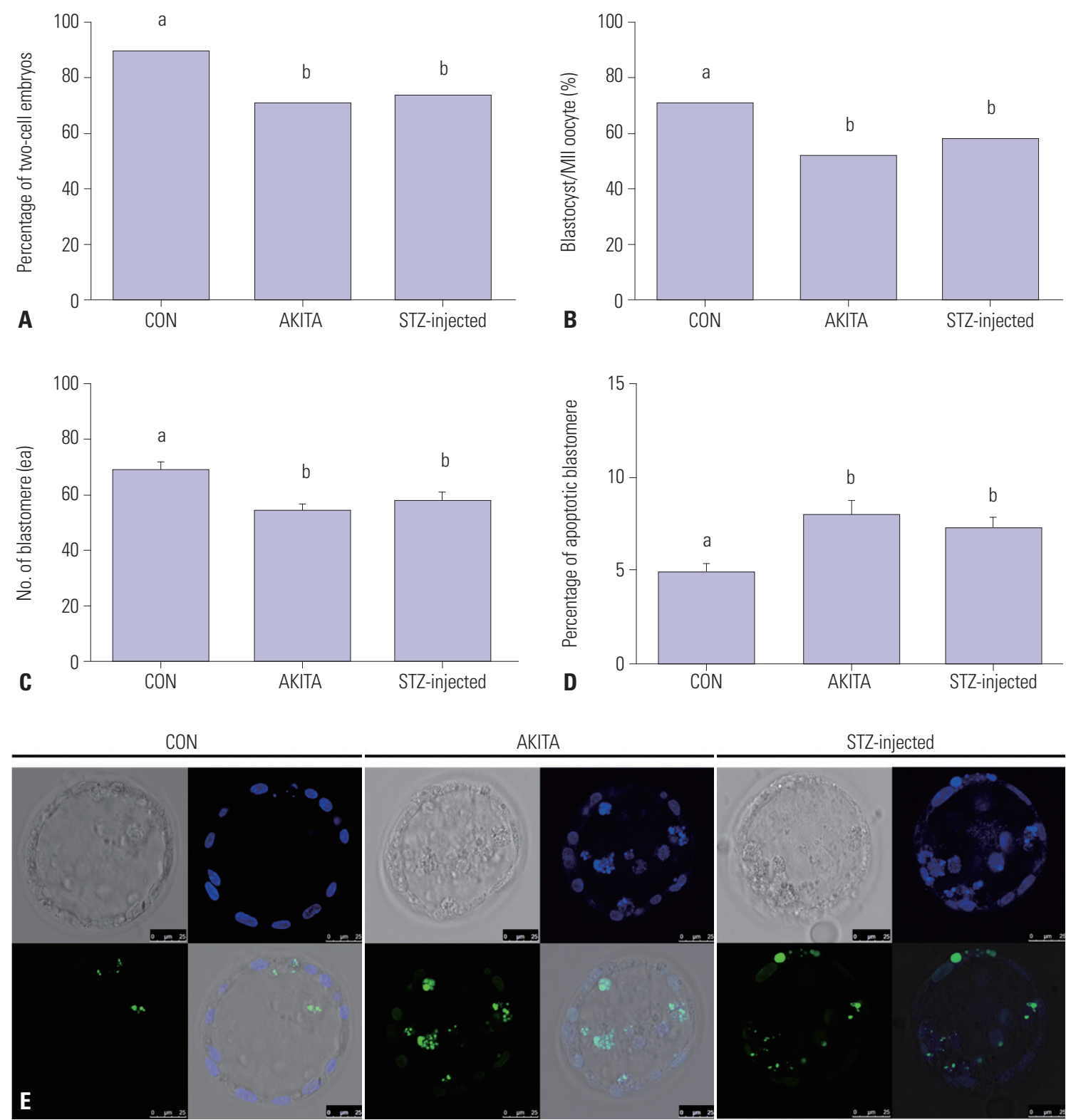

Fig. 6. Embryonic developmental competence in type I diabetes mellitus mouse models was significantly lower compared with normal control mice. ( $A$ and B) Percentages of cleavage and blastulation after in vitro fertilization (IVF). The numbers of two-cell embryos and blastocysts were counted to compare pre-implantation embryonic development $(n=85)$. ( $C$ and D) Total and apoptotic blastomeres of individual blastocysts were also evaluated by confocal microscopy ( $n=21)$. (E) Representative image of a hatching blastocyst after staining with 2-(4-amidinophenyl)-1H -indole-6-carboxamidine (DAPI) and terminal deoxynucleotidyl transferase dUTP nick end-labeling (TUNEL). Magnification was $\times 200$ and the images were obtained by confocal microscopy. Blue and Green signal respectively indicate nucleus and apoptotic DNA of blastomeres. Different letters $(a, b)$ indicate statistically significant differences $(p<0.05)$. STZ, streptozotocin; CON, control; MII, metaphase II.

quality. Our study is limited in that it compared only two T1D mouse models and that exogenous insulin treatment was not used to examine factors underlying differences in reproductive function between the models. Future mechanistic studies of the effects of T1D on reproductive function may provide additional insights that may improve reproductive outcomes in women with T1D.

\section{ACKNOWLEDGEMENTS}

Plasmid DNA for the qPCR standard curve used in this study was kindly provided by Dr. Rebecca Robker. The MSY2 antibody used in this study for immunohistochemistry was kindly provided by Dr. Richard Schultz.

This study was funded by grants P50 HD076188 and UG3 ES029073 from the NIH and the Basic Science Research Program through the National Research Foundation of Korea (NRF), funded by the Ministry of Education (NRF-2018R1D- 


\section{A1B07046419).}

\section{AUTHOR CONTRIBUTIONS}

Jaewang Lee performed the research and wrote the manuscript. Hoi Chang Lee performed research and conducted data analyses. So-Youn Kim contributed to discussion and reviewed/edited manuscript. Geum Joon Cho contributed to discussion and reviewed/edited manuscript. Teresa K. Woodruff reviewed and edited the manuscript.

\section{ORCID iDs}

Jaewang Lee Hoi Chang Lee So-Youn Kim https://orcid.org/0000-0001-6801-7149 Geum Joon Cho https://orcid.org/0000-0002-6436-8918 https://orcid.org/0000-0003-1013-6852 Teresa K. Woodruff https://orcid.org/0000-0002-1197-3399

\section{REFERENCES}

1. Codner E, Merino PM, Tena-Sempere M. Female reproduction and type 1 diabetes: from mechanisms to clinical findings. Hum Reprod Update 2012;18:568-85.

2. Clark M, Kroger CJ, Tisch RM. Type 1 diabetes: a chronic anti-selfinflammatory response. Front Immunol 2017;8:1898.

3. Gerard-Gonzalez A, Gitelman SE, Cheng P, Dubose SN, Miller $\mathrm{KM}$, Olson BA, et al. Comparison of autoantibody-positive and autoantibody-negative pediatric participants enrolled in the T1D Exchange clinic registry. J Diabetes 2013;5:216-23.

4. Abell SK, Nankervis A, Khan KS, Teede HJ. Type 1 and Type 2 diabetes preconception and in pregnancy: health impacts, influence of obesity and lifestyle, and principles of management. Semin Reprod Med 2016;34:110-20.

5. Codner E, Eyzaguirre FC, Iñiguez G, López P, Pérez-Bravo F, Torrealba IM, et al. Ovulation rate in adolescents with type 1 diabetes mellitus. Fertil Steril 2011;95:197-202.

6. Chang AS, Dale AN, Moley KH. Maternal diabetes adversely affects preovulatory oocyte maturation, development, and granulosa cell apoptosis. Endocrinology 2005;146:2445-53.

7. Diamond MP, Moley KH, Pellicer A, Vaughn WK, DeCherney AH. Effects of streptozotocin- and alloxan-induced diabetes mellitus on mouse follicular and early embryo development. J Reprod Fertil 1989;86:1-10.

8. Ratchford AM, Chang AS, Chi MM, Sheridan R, Moley KH. Maternal diabetes adversely affects AMP-activated protein kinase activity and cellular metabolism in murine oocytes. Am J Physiol Endocrinol Metab 2007;293:E1198-206.

9. Wang Q, Frolova AI, Purcell S, Adastra K, Schoeller E, Chi MM, et al. Mitochondrial dysfunction and apoptosis in cumulus cells of type I diabetic mice. PLoS One 2010;5:e15901.

10. Wang J, Takeuchi T, Tanaka S, Kubo SK, Kayo T, Lu D, et al. A mutation in the insulin 2 gene induces diabetes with severe pancreatic beta-cell dysfunction in the Mody mouse. J Clin Invest 1999;103: 27-37.

11. Yamane S, Hamamoto Y, Harashima S, Harada N, Hamasaki A, Toyoda K, et al. GLP-1 receptor agonist attenuates endoplasmic reticulum stress-mediated $\beta$-cell damage in Akita mice. J Diabetes Investig 2011;2:104-10.

12. Jo H, Byun HM, Lee SI, Shin DM. Initiation site of $\mathrm{Ca}(2+)$ entry evoked by endoplasmic reticulum $\mathrm{Ca}(2+)$ depletion in mouse parotid and pancreatic acinar cells. Yonsei Med J 2007;48:526-30.

13. Lee KG, Yoo MS, Choi IJ. The effect of corticosteroid on the fetal pulmonary maturation of rats with streptozotocin-induced diabetes. Yonsei Med J 1986;27:121-31.

14. Deeds MC, Anderson JM, Armstrong AS, Gastineau DA, Hiddinga HJ, Jahangir A, et al. Single dose streptozotocin-induced diabetes: considerations for study design in islet transplantation models. Lab Anim 2011;45:131-40.

15. Xu M, Banc A, Woodruff TK, Shea LD. Secondary follicle growth and oocyte maturation by culture in alginate hydrogel following cryopreservation of the ovary or individual follicles. Biotechnol Bioeng 2009;103:378-86.

16. Choi YS, Song JE, Kong BS, Hong JW, Novelli S, Lee EJ. The role of Foxo3 in Leydig cells. Yonsei Med J 2015;56:1590-6.

17. Barber AJ, Antonetti DA, Kern TS, Reiter CE, Soans RS, Krady JK, et al. The Ins2Akita mouse as a model of early retinal complications in diabetes. Invest Ophthalmol Vis Sci 2005;46:2210-8.

18. Kim SY, Ebbert K, Cordeiro MH, Romero M, Zhu J, Serna VA, et al. Cell autonomous phosphoinositide 3-kinase activation in oocytes disrupts normal ovarian function through promoting survival and overgrowth of ovarian follicles. Endocrinology 2015;156:1464-76.

19. Kim SE, Koo JS, Jung WH. Immunophenotypes of glycogen rich clear cell carcinoma. Yonsei Med J 2012;53:1142-6.

20. Kim EJ, Lee J, Youm HW, Kim SK, Lee JR, Suh CS, et al. Comparison of follicle isolation methods for mouse ovarian follicle culture in vitro. Reprod Sci 2018;25:1270-8.

21. Mainigi MA, Ord T, Schultz RM. Meiotic and developmental competence in mice are compromised following follicle development in vitro using an alginate-based culture system. Biol Reprod 2011; 85:269-76.

22. Richani D, Sutton-McDowall ML, Frank LA, Gilchrist RB, Thompson JG. Effect of epidermal growth factor-like peptides on the metabolism of in vitro- matured mouse oocytes and cumulus cells. Biol Reprod 2014;90:49.

23. Wiebe JC, Santana A, Medina-Rodríguez N, Hernández M, Nóvoa J, Mauricio D, et al. Fertility is reduced in women and in men with type 1 diabetes: results from the Type 1 Diabetes Genetics Consortium (T1DGC). Diabetologia 2014;57:2501-4.

24. Wu LL, Russell DL, Wong SL, Chen M, Tsai TS, St John JC, et al. Mitochondrial dysfunction in oocytes of obese mothers: transmission to offspring and reversal by pharmacological endoplasmic reticulum stress inhibitors. Development 2015;142:681-91.

25. Gordo AC, Kurokawa M, Wu H, Fissore RA. Modifications of the $\mathrm{Ca} 2+$ release mechanisms of mouse oocytes by fertilization and by sperm factor. Mol Hum Reprod 2002;8:619-29.

26. Sutton-McDowall ML, Wu LL, Purdey M, Abell AD, Goldys EM, MacMillan KL, et al. Nonesterified fatty acid-induced endoplasmic reticulum stress in cattle cumulus oocyte complexes alters cell metabolism and developmental competence. Biol Reprod 2016;94:23.

27. Zhang N, Duncan FE, Que EL, O'Halloran TV, Woodruff TK. The fertilization-induced zinc spark is a novel biomarker of mouse embryo quality and early development. Sci Rep 2016;6:22772.

28. Lee J, Kim J, Kim SH, Kang HG, Jun JH. Effects of coculture with immune cells on the developmental competence of mouse preimplantation embryos in vitro and in utero. Reprod Sci 2015;22:125261.

29. Chillarón JJ, Benaiges D, Mañé L, Pedro-Botet J, Flores Le-Roux JA. Obesity and type 1 diabetes mellitus management. Minerva Endocrinol 2015;40:53-60.

30. Schoeller EL, Albanna G, Frolova AI, Moley KH. Insulin rescues impaired spermatogenesis via the hypothalamic-pituitary-gonadal axis in Akita diabetic mice and restores male fertility. Diabetes 2012;61:1869-78.

31. Schoeller EL, Chi M, Drury A, Bertschinger A, Esakky P, Moley $\mathrm{KH}$. Leptin monotherapy rescues spermatogenesis in male Akita 
type 1 diabetic mice. Endocrinology 2014;155:2781-6.

32. Johnson LM, Sidman RL. A reproductive endocrine profile in the diabetes (db) mutant mouse. Biol Reprod 1979;20:552-9.

33. Wang Q, Chi MM, Moley KH. Live imaging reveals the link between decreased glucose uptake in ovarian cumulus cells and impaired oocyte quality in female diabetic mice. Endocrinology 2012;153:1984-9.

34. Wang Q, Moley KH. Maternal diabetes and oocyte quality. Mitochondrion 2010;10:403-10.

35. Burkart AD, Xiong B, Baibakov B, Jiménez-Movilla M, Dean J. Ovastacin, a cortical granule protease, cleaves ZP2 in the zona pellucida to prevent polyspermy. J Cell Biol 2012;197:37-44.

36. Xiong B, Zhao Y, Beall S, Sadusky AB, Dean J. A unique egg cortical granule localization motif is required for ovastacin sequestration to prevent premature ZP2 cleavage and ensure female fertili- ty in mice. PLoS Genet 2017;13:e1006580.

37. Wai T, Ao A, Zhang X, Cyr D, Dufort D, Shoubridge EA. The role of mitochondrial DNA copy number in mammalian fertility. Biol Reprod 2010;83:52-62.

38. Zhang CH, Qian WP, Qi ST, Ge ZJ, Min LJ, Zhu XL, et al. Maternal diabetes causes abnormal dynamic changes of endoplasmic reticulum during mouse oocyte maturation and early embryo development. Reprod Biol Endocrinol 2013;11:31.

39. Moley KH, Chi MM, Mueckler MM. Maternal hyperglycemia alters glucose transport and utilization in mouse preimplantation embryos. Am J Physiol 1998;275:E38-47.

40. Chi MM, Hoehn A, Moley KH. Metabolic changes in the glucoseinduced apoptotic blastocyst suggest alterations in mitochondrial physiology. Am J Physiol Endocrinol Metab 2002;283:E226-32. 